\title{
Alimentos ricos em triptofano e seu efeito na liberação da serotonina e possíveis benefícios no transtorno de ansiedade
}

\author{
Foods rich in tryptophan and its effect on serotonin release and possible benefits in anxiety
} disorder

Alimentos ricos en triptófano y su efecto en la liberación de serotonina y posibles beneficios en el trastorno de ansiedad

\author{
Dourival Tavares Sousa Júnior \\ ORCID: https://orcid.org/0000-0001-7525-6535 \\ Centro Universitário Santo Agostinho, Brasil \\ E-mail: dourivaltsousa@gmail.com \\ Thiago Ferreira Cândido Lima Verde \\ ORCID: https://orcid.org/0000-0002-1602-087X \\ Universidade Federal do Piauí, Brasil \\ E-mail: thiagovalantysor@gmail.com \\ Liejy Agnes Santos Raposo Landim \\ ORCID: https://orcid.org/0000-0002-8214-2832 \\ Centro Universitário Santo Agostinho, Brasil \\ E-mail: liejyagnes@gmail.com
}

\begin{abstract}
Resumo
A serotonina, ou 5-hidroxitriptamina, é uma monoamina neurotransmissora no sistema nervoso central da maioria dos animais, incluindo seres humanos. Sua síntese depende do fornecimento do aminoácido essencial, L-triptofano, que não pode ser biossintetizado pelo ser humano e, portanto, deve ser obtido a partir de fontes dietéticas. O presente estudo tem como objetivo discutir alimentos ricos em triptofano na liberação da serotonina e seus possíveis benefícios no transtorno de ansiedade. Foi realizado um levantamento de dados no Google Acadêmico, PubMed, LiLacs, Scielo e CAPES onde os critérios de inclusão foram artigos disponíveis na integra online em língua portuguesa e inglesa publicados entre os anos de 2011e 2021 para as pesquisas mais recentes, bem como outras pesquisas sobre o assunto de forma atemporal e os critérios de exclusão foram estudos que não estavam na integra e que não abordavam de forma clara o assunto requerido. Os alimentos ricos em triptofano ajudam na síntese e no controle da serotonina no organismo, sendo responsável por nos prover sensação de bem-estar. Suprimentos ricos nesse aminoácido incluem: banana, ovo, leite, chocolate amargo, amêndoas, mel, sementes e grãos. A 5-HT gerada a partir do triptofano, tem efeito em várias funções do Sistema Nervoso Central e em vários processos no organismo, nomeadamente na regulação do humor, do stress e da agressividade. Além disso, baixos níveis podem ocasionar psicopatologias como a ansiedade. Com o propósito de auxiliar, inserir alimentos ricos em triptofano pode melhorar sintomas físicos e mentais visto que, a serotonina tem um papel muito importante nesses processos.
\end{abstract}

Palavras-chave: Alimento funcional; Ansiedade; Triptofano; Serotonina; Receptores serotonérgicos.

\begin{abstract}
Serotonin, or 5-hydroxytryptamine, is a monoamine neurotransmitter in the central nervous system of most animals, including humans. Its synthesis depends on the supply of the essential amino acid, L-tryptophan, which cannot be biosynthesized by humans and therefore must be obtained from dietary sources. This study aims to discuss foods rich in tryptophan in serotonin release and its possible benefits in anxiety disorder. A data survey was conducted in Google Scholar, Pubmed, Lilacs, Scielo and CAPES where the inclusion criteria were articles available online in Portuguese and English published between the years 2011 and 2021 for the most recent research, as well as other research on the subject in a timeless way and the exclusion criteria were studies that were not in full and that did not clearly address the required subject. Foods rich in tryptophan help in the synthesis and control of serotonin in the body, being responsible for providing us with a feeling of well-being. Supplies rich in this amino acid include: banana, egg, milk, bitter chocolate, almonds, honey, seeds and grains. The 5-HT generated from tryptophan has an effect on various functions of the Central Nervous System and on various processes in the body, namely the regulation of mood, stress and aggressiveness. In addition, low levels can cause psychopathologies such as anxiety. For the purpose of helping, inserting foods rich in tryptophan can improve physical and mental symptoms since serotonin plays a very important role in these processes.
\end{abstract}

Keywords: Functional food; Anxiety; Tryptophan; Serotonin; Serotonergic receptors. 


\begin{abstract}
Resumen
La serotonina, o 5-hidroxitriptamina, es una monoamina neurotransmisora en el sistema nervioso central de la mayoría de los animales, incluidos los seres humanos. Su síntesis depende del suministro del aminoácido esencial, Ltriptófano, que no puede ser biosintetizado por el ser humano y por lo tanto debe obtenerse de fuentes dietéticas. El presente estudio tiene como objetivo discutir alimentos ricos en triptófano en la liberación de la serotonina y sus posibles beneficios en el trastorno de ansiedad. Se realizó un levantamiento de datos en Google Académico, PubMed, Lilacs, Scielo y CAPES donde los criterios de inclusión fueron artículos disponibles en la integra online en lengua portuguesa e inglesa publicados entre los años 2011 y 2021 para las investigaciones más recientes, así como otras investigaciones sobre el asunto de forma atemporal y los criterios de exclusión fueron estudios que no estaban en la integra y que no abordaban de forma clara el asunto requerido. Los alimentos ricos en triptófano ayudan en la síntesis y el control de la serotonina en el cuerpo, siendo responsable de proporcionarnos sensación de bienestar. Los suministros ricos en este aminoácido incluyen: plátano, huevo, leche, chocolate amargo, almendras, miel, semillas y granos. La 5-HT generada a partir del triptófano, tiene efecto en varias funciones del Sistema Nervioso Central y en varios procesos en el organismo, en particular en la regulación del humor, del estrés y de la agresividad. Además, los niveles bajos pueden ocasionar psicopatologías como la ansiedad. Con el propósito de ayudar, insertar alimentos ricos en triptófano puede mejorar los síntomas físicos y mentales ya que, la serotonina tiene un papel muy importante en estos procesos.
\end{abstract}

Palabras clave: Alimento funcional; Ansiedad; Triptófano; Serotonina; Receptores serotonérgicos.

\title{
1. Introdução
}

A alimentação é uma necessidade biológica para a nossa sobrevivência, entretanto, os valores culturais e simbólicos são agregados à alimentação dos humanos determinando seu comportamento alimentar, o qual é originado por aspectos demográficos, econômicos, sociais, culturais, ambientais, psicológicos e nutricionais de um indivíduo ou sociedade (Morais, 2014).

A serotonina, ou 5-hidroxitriptamina, é uma monoamina neurotransmissora no sistema nervoso central da maioria dos animais, incluindo seres humanos. Sua síntese depende do fornecimento do aminoácido essencial, L-triptofano, que não pode ser biossintetizado pelo ser humano e, portanto, deve ser obtido a partir de fontes dietéticas. Em alguns alimentos naturais é possível encontrar o triptofano, como por exemplo: bananas, semente de abóbora, soja, grão-de-bico, tâmaras secas, amendoins, leite, carne, peixe, peru e alimentos ricos em proteínas. Além disso, a taxa de síntese de serotonina depende da disponibilidade do precursor triptofano. Uma consideração importante para o entendimento dos efeitos da administração do triptofano é que apenas cerca de 5\% da serotonina endógena é encontrada no cérebro; o restante está no intestino (cerca de 90\%), liberado principalmente pelas células enterocromafins, e no tecido periférico ou no sangue, onde é absorvido pelas plaquetas sanguíneas (Fernstrom, 2016; Jenkins, et al., 2016; Abreu, 2021).

O nome serotonina deriva de sua descoberta no sangue há 70 anos e da observação de que causava a contração do músculo liso vascular (Watts, et al., 2012); assim, uma função da serotonina é regular o fluxo sanguíneo local. Essa distribuição desequilibrada entre o cérebro e a periferia deve ser levada em consideração quanto ao possível impacto da manipulação dietética da serotonina central pelo triptofano. Essas questões são consideradas posteriormente; no entanto, a serotonina é um neurotransmissor do sistema nervoso central amplamente distribuído e importante, originando-se de corpos celulares neuronais localizados nos núcleos superior e inferior da rafe do tronco encefálico e atuando em múltiplos subtipos de receptores com uma gama de efeitos comportamentais como os responsáveis pela ansiedade, medo, depressão, sono e percepção à dor. (Jacobs \& Martin-cora \& Fornal, 2002).

A importância estabelecida da serotonina nos transtornos afetivos e no apetite, bem como no sono e na cognição (Fernstrom, et al., 2013), torna a compreensão de quem pode se beneficiar mais do uso terapêutico do triptofano um importante objetivo de pesquisa. Nesta perspectiva, o presente estudo tem como objetivo discutir alimentos ricos em triptofano na liberação da serotonina e seus possíveis benefícios no transtorno de ansiedade. 


\section{Metodologia}

A pesquisa será descritiva do tipo revisão narrativa da literatura, onde não se objetiva saturar os achados e nem mesmo os entremeios que envolvem a temática, com estudo de artigos e livros. Tal revisão viabiliza apontar lacunas em determinada área no conhecimento e realizar a síntese de múltiplos estudos publicados, permitindo conclusões gerais a respeito do assunto em estudo (Mendes, et al., 2008).

O presente estudo trata-se de uma revisão narrativa da literatura de abordagem quantitativa, com objetivo descritivo e exploratório, que utiliza dados por meio de consultas em artigos científicos já publicados que são considerados relevantes. A pesquisa foi composta por estudos científicos que englobavam alimentos ricos em triptofano e a libração da serotonina levando em consideração seus possíveis benefícios no transtorno da ansiedade. Sendo motivado pelo seguinte questionamento: Alimentos ricos em triptofano e seu efeito na liberação da serotonina tem algum possível benefício no transtorno de ansiedade?

Primeiramente, foi realizado um levantamento de dados no Google Acadêmico, PubMed, LiLacs, Scielo e CAPES onde os critérios de inclusão foram artigos disponíveis na integra online em língua portuguesa e inglesa publicados entre os anos de 2011e 2021 para as pesquisas mais recentes, bem como outras pesquisas sobre o assunto de forma atemporal sobre o tema de alimentos ricos em triptofano e seu efeito na liberação da serotonina e possíveis benefícios para a saúde. E os critérios de exclusão foram estudos que não estavam na integra e que não abordavam de forma clara o assunto requerido.

Nas buscas, os descritores utilizados na base de dados da PubMed, LiLacs, Scielo, CAPES e Google Acadêmico foram mediante o cruzamento dos seguintes descritores: "Triptofano", "Serotonina", "Alimento Funcional", "Ansiedade", "Receptores Serotonérgicos" e suas respectivas traduções para o inglês: "Tryptophan", "Serotonin", "Functional Food", "Anxiety" e "Serotonergic Receptors" cadastrados nos Descritores em Ciências da Saúde (DeCS) sendo combinados pelo operador booleano "AND", a fim de localizar os registros de interesse.

As informações extraídas dos artigos selecionados foram agrupadas em temas afins, onde foram observadas às informações sobre as metodologias utilizadas, os resultados alcançados e as conclusões a que os autores chegaram. Realizaram-se as buscas iniciais pelos resumos dos artigos que respondam aos descritores adotados e, foram selecionados aqueles que mencionaram fatores relacionados aos alimentos ricos em triptofano e seu efeito na liberação da serotonina e seus possiveis beneficios no transtorno de ansiedade. A partir de repetidas leituras dos resumos selecionados na fase anterior, foram extraídos aqueles estudos que versaram a respeito dos efeitos do triptofano presente nos alimentos na liberação da serotonina e seus possiveis beneficios no transtorno de ansiedade.

\section{Resultados e Discussão}

\subsection{Triptofano e seu efeito na liberação da serotonina}

A serotonina, ou 5-HT (5-hidroxitriptamina), é um neurotransmissor de monoamina, que atua em receptores de membrana no sistema nervoso central (SNC) e no sistema nervoso periférico (SNP), bem como em tecidos não neuronais (sangue, gastrointestinal, endócrino, sistemas sensoriais e cardiovasculares). Por ter características hidrofílicas, a serotonina não é capaz de permear a barreira hematoencefálica e, portanto, sua síntese se dá a partir do aminoácido triptofano nos neurônios do núcleo da rafe mediana mesencefálica (Lima, et al., 2020).

O triptofano, um aminoácido essencial, é o único precursor da serotonina e sua concentração plasmática é determinada pelo balanço entre a ingestão dietética e sua remoção do plasma para síntese proteica (Sezini, et al., 2014).

Após ser absorvido pelo intestino, fica disponível na circulação (onde existe como fração livre ou ligada à albumina) e atravessa a barreira hematoencefálica (BHE) através do transportador dos aminoácidos neutros, a fim de participar na síntese de 5-HT no sistema nervoso central. O triptofano, no neurónio serotoninérgico, é convertido em 5-hidroxi-triptofano (5-HTP) 
por meio da enzima triptofano hidroxilase (TPH), sendo este o passo limitante na sua síntese, e posteriormente convertido em 5-HT através do Laminoácido-aromático-descarboxilase (AAAD) (Santos, 2016).

O triptofano é absorvido por neurônios serotoninérgicos e convertidos em serotonina, que é armazenada nas vesículas sinápticas. A estimulação dos neurônios faz com que as vesículas se fundam com a membrana neuronal e liberar serotonina na sinapse. A serotonina liberada se liga aos receptores na fase pós-sináptica neurônios que levam à ativação de vias de sinalização e respostas. A ação da serotonina é encerrada por sua difusão da sinapse e metabolismo subsequente, ou por recaptação de volta para o pré-sináptico neurônio por um transportador de recaptação de serotonina para armazenamento ou metabolismo (Seitz, et al., 2018)

A 5-HT gerada a partir do triptofano, tem efeito em várias funções do Sistema Nervoso Central (SNC) e em vários processos no organismo, nomeadamente na regulação do humor, do stress, da agressividade, do comportamento alimentar, da temperatura corporal, da cognição, do sistema cardiovascular e do comportamento sexual. Além disso, baixos níveis podem acarretar psicopatologias como a ansiedade (Santos, 2016; Zanello, 2012).

De acordo com Araujo et al., (2020) a absorção adequada desse aminoácido e de nutrientes como magnésio, e vitaminas do complexo B, é essencial no tratamento da depressão, pois a quantidade de 5-HT sintetizada está diretamente relacionada à biodisponibilidade de triptofano plasmático e da atividade da enzima triptofano hidroxilase.

Lindseth et al., (2015) indicaram que o aumento de triptofano na ingestão dietética (10 mg/kg), resultou em menos depressão, ansiedade e melhora no humor quando comparado a um baixo consumo na dieta ( $5 \mathrm{mg} / \mathrm{kg}$ ). Sugerindo que os níveis de neurotransmissores de serotonina no cérebro podem estar relacionados à quantidade de triptofano ofertada na alimentação.

Segundo Andrade et al., (2018) um estudo clínico randomizado, constatou que a administração via oral da associação do L-triptofano, ômega 3, magnésio e vitaminas do complexo B se mostrou tão eficiente quanto a administração do ômega 3 na redução dos sintomas da ansiedade na amostra estudada.

\subsection{Alimentos ricos em triptofano}

De modo geral, os hábitos alimentares saudáveis são importantes para manutenção do quadro global de saúde do indivíduo (Tavares, et al., 2014). Torna-se cada vez mais aceito que a ingestão de alimentos afeta o humor e, ainda mais, o humor parece influenciar na decisão de que tipo de alimentos preferimos (Strasser, et al., 2016).

De acordo com Zanello (2012) os neurotransmissores correspondem a substâncias químicas mensageiras que enviam sinais pela fenda sináptica entre as células nervosas encontradas em todo o corpo humano. Os neurotransmissores são formados a partir de aminoácidos, vitaminas e cofatores minerais. A maioria dos neurotransmissores do Sistema Nervoso Central (SNC) é sintetizada a partir de aminoácidos que são adquiridos pela dieta.

O triptofano é um aminoácido essencial que serve a vários propósitos importantes, como equilíbrio de nitrogênio em adultos e crescimento em bebês. Também é usado para produzir niacina, que é essencial para a criação do neurotransmissor serotonina (Abreu, 2021).

O triptofano é um aminoácido que o organismo humano não produz, sendo adquirido por meio de dieta (Lima, et al., 2018). É através do meio externo, ou seja, da alimentação, que o suprimento triptofano pode ser alcançado. Existem vários alimentos que são ricos nesse aminoácido, os quais, quando ingeridos, ajudam na síntese e no controle da serotonina no organismo, sendo responsável por nos prover sensação de bem estar. Suprimentos ricos nesse aminoácido incluem: banana, ovo, leite, chocolate amargo, queijo, amêndoas, arroz integral, mel, nozes, sementes e grãos (de Oliveira, et al., 2019; Machado, et al., 2018; Strasser, et al., 2016).

Na Tabela 1 se encontra a quantidade de triptofano por 100 gramas em alimentos comuns. 
Tabela 1 - Quantidade de triptofano por $100 \mathrm{~g}$ em alimentos comuns.

\begin{tabular}{|c|c|}
\hline ALIMENTO (100 gramas & TRIPTOFANO (miligramas) \\
\hline Leite & 42 \\
\hline Ovos & 165 \\
\hline Banana & 10 \\
\hline Chocolate & 290 \\
\hline
\end{tabular}

Fonte: Adaptado de Fernstrom (2016).

O leite contém triptofano que é um aminoácido essencial para a produção de serotonina pelo cérebro e que regula áreas cerebrais responsáveis pelo comportamento alimentar, humor, vício e depressão, além de ajudar a melhorar o desempenho do cérebro e memória, e ajudar a ter um sono mais tranquilo, o que é fundamental para armazenar a informação aprendida (Ferraz, 2021).

O ovo é uma boa fonte de nutrientes relacionados à saúde do cérebro, incluindo vitaminas B6 e B12, folato e colina. As vitaminas do complexo B e o ácido fólico são essenciais para o desenvolvimento do cérebro e para formação dos componentes dos neurônios, melhorando o seu funcionamento. Além de seu papel como um cofator necessário no ciclo do folato, o papel da vitamina B6 no metabolismo de aminoácidos torna um cofator limitante na síntese de neurotransmissores como dopamina, serotonina, ácido $\gamma$-aminobutírico (GABA), noradrenalina e o hormônio melatonina. Além disso, a falta de vitamina B6 impede a utilização pelo organismo do triptofano para a síntese de serotonina (Ferraz, 2021; da Fonseca Freitas, et al, 2021; Kennedy, 2016).

As bananas contêm carboidratos complexos, macronutrientes associados com transtornos de ansiedade. Hidratos de carbono complexos com um baixo índice glicêmico e contêm fibras irá aumentar os níveis de serotonina, um produto químico no cérebro que faz acalmar e estabiliza a pressão arterial como uma maneira de abrandar o estresse e transtornos de ansiedade. Triptofano contido em bananas é um precursor da síntese de serotonina, foi encontrado para melhorar o humor, reduzir os transtornos de ansiedade e depressão. O hormônio insulina, que é desencadeada através do consumo de carboidratos, pode aumentar a síntese de triptofano, que, em última análise, estimula a síntese de neurotransmissores importantes, incluindo a serotonina (Setyarini, et al., 2020).

O chocolate preto se bem adequado na dieta pode ser de fundamental importância na melhora dos sintomas do Sistema Nervoso Central (SNC) por conter alguns aminoácidos essenciais como triptofano que serve como percursor para a serotonina, conhecida como o hormônio da felicidade. A falta deste aminoácido pode manifestar depressão ou estados ansiosos no indivíduo. Ressaltando que, para desfrutar dos benefícios do cacau, sugere-se o consumo de chocolates com ao menos 70\% de cacau (da Fonseca Freitas, et al, 2021; de Oliveira, et al, 2019; Ferraz, 2021).

\subsection{Serotonina e seus possíveis benefícios no transtorno de ansiedade}

Estudos tem cada vez mais evidenciados que a fisiopatologia desses transtornos tem relação com disfunção de neurotransmissores, sendo eles responsáveis pela comunicação química do cérebro. Um exemplo é a serotonina 5-HT, produzida pelo aminoácido triptofano, que tem papel de fundamental importância, pois o sistema serotoninérgico atua na regulação dos processos de comportamento e emoções, na modulação e motilidade gastrointestinal, regulação hidroeletrolítica, balanço energético e outros níveis baixos de serotonina podem contribuir para aumentar ansiedade e depressão (dos Anjos, et al., 2020).

Segundo Araujo et. al., (2020) os transtornos de ansiedade e depressão constituem um grande impasse de saúde pública estando como uma das principais causas de incapacitação do mundo limitando a capacidade física, mental e social. No 
contexto da qualidade de vida, destaca-se a alimentação dado que uma dieta inadequada está relacionada com ansiedade intensa podendo desencadear transtornos como anorexia nervosa, bulimia, e obesidade, causando prejuízo ao indivíduo.

Uma boa saúde mental e bem-estar podem estar relacionados à disponibilidade de serotonina, que é dependente da síntese de triptofano, um aminoácido essencial obtido por fontes alimentares (da Rocha, et al., 2020). A neurotransmissão serotoninérgica é muito complexa e está envolvida na regulação do humor, controle de impulsos, sono, vigilância, alimentação, libido, memória e aprendizagem. As deficiências do sistema serotonérgico causam distúrbios como depressão, fobias, estresse pós-traumático e transtorno da ansiedade generalizada (Lima, et al., 2020).

Os estados de ansiedade e depressão podem estar relacionados a uma dieta inflamatória, com o inadequado consumo de alimentos ricos em açúcar, gorduras, pouca ingestão de frutas e vegetais, logo, um padrão de alimentação saudável com compostos bioativos pode ter um efeito protetor no tratamento dessas patologias (Jacka, et al., 2015). Segundo Lindseth et al. (2015) existe uma relação dos efeitos do triptofano, um aminoácido precursor da serotonina, com a regulação do humor e da ansiedade, uma vez que os baixos níveis de serotonina cerebral podem contribuir para aumentar a ansiedade e a depressão.

Neste contexto alimentos como os ovos, leites e seus derivados são alimentos ricos em nutrientes como o triptofano que produz o efeito de relaxar a musculatura, além de conter substâncias que ajudam no bom humor como as vitaminas do complexo B (tiamina, piridoxina, riboflavina, niacina e cobalamina) e ajudando em conjunto a memória. Os nutrientes mais encontrados nesses alimentos, além das proteínas e aminoácidos (tirosina), são cálcio, ferro, cobre, cromo, zinco, fósforo, vitaminas A (retinol) e D (calciferol) e a biotina (Tavares, et al., 2014).

Por fim, ficou evidente a relação favorável que alimentos ricos em triptofano na liberação da serotonina trazem benefícios para o transtorno de ansiedade, ressaltando assim, a necessidade da inclusão de alimentos ricos em triptofano para prevenção e/ou recuperação de diversas doenças físicas e mentais.

\section{Considerações Finais}

Portanto, através dos resultados expostos ficou claro a importância de se consumir alimentos ricos em triptofano para que o seu efeito na liberação da serotonina no corpo traga seus devidos benefícios, como por exemplo, na depressão, ansiedade, humor, controle de impulsos, sono, vigilância, alimentação, libido, memória e aprendizagem e que quando estão em deficiência o corpo manifesta diversos sinais e sintomas na saúde física e mental. Com o propósito de auxiliar, inserir alimentos ricos em triptofano pode melhorar sintomas físicos e mentais visto que, a serotonina tem um papel muito importante nesses processos.

Apesar de relevância do efeito do triptofano e seu efeito na liberação da serotonina e seus possíveis benefícios no transtorno de ansiedade, o presenta estudo abre lacunas para pesquisas posteriores de larga escala acerca do efeito da serotonina sobre o transtorno de ansiedade, para entender como esse processo ocorre, assim como, ele age para a melhora de pessoas acometidas com transtorno, contribuindo para melhores resultados no tratamento e proporcionando uma melhor qualidade de vida ao paciente.

\section{Referências}

Abreu, F. (2021). Detalhamento sobre o triptofano e sua importância no combate aos agentes que levam à depressão: Avaliação sobre o produto Triptolife: Details about tryptophan and its importance in combating agents that lead to depression: Evaluation of the triptolife product. CPAH Scientific Journal of Health, 1(3), 130-141.

Araújo, A. D. S. F., Vieira, I. N. U., Silva, J. N. F. D., Faria, S. P. D., Nunes, G. L., Khouri, A. G., \& Silveira, A. A. D. (2020). Avaliação do consumo alimentar em pacientes com diagnóstico de depressão e/ou ansiedade. Referências em Saúde da Faculdade Estácio de Sá de Goiás-RRS-FESGO, 3(1).

da Fonseca Freitas, F., Santos, J. C. C., de Medeiros, A. C. Q., \& de Araújo Lopes, F. (2021). Desenvolvimento de cartilha sobre os benefícios da alimentação para reduzir a ansiedade em tempos de COVID-19: Relato de experiência. Revista Brasileira de Extensão Universitária, 12(2), $257-267$. 
da Rocha, A. C. B., Myva, L. M. M., \& de Almeida, S. G. (2020). O papel da alimentação no tratamento do transtorno de ansiedade e depres são. Research, Society and Development, 9(9), e724997890-e724997890.

da Silva Lima, L., \& da Silva, C. P. (2018). Triptofano no Sono: Uma Revisão Sistemática baseada no Método PRISMA. ID on line Revista De Psicologia, 12(42), 397-407.

de Andrade, E. A. F., Sant'Anna, L. C., de Castro Almeida, N., Venturi, I., Brustulim, L. J. R., \& D’Almeida, W. O. (2018). L-Triptofano, ômega 3, magnésio e vitaminas do complexo B na diminuição dos sintomas de ansiedade. Id on Line Revista de Psicologia, 12(40), 1129-1138.

de Matos Feijó, F., Bertoluci, M. C., \& Reis, C. (2011). Serotonina e controle hipotalâmico da fome: uma revisão. Revista da Associação Médica Brasileira, 57(1), 74-77.

de Oliveira, A. P. A., Moleiro, I. C. G., dos Santos Pessoa, S. C., Caldeira, J. E. B., \& da Costa, E. S. (2019). Os Alimentos E Os Transtornos Mentais.

dos Anjos, A. S., da Costa, C. M. F. P., de Moraes, C. T. V., Aquino, C. C., Fametro-Unifametro, D. C. U., Científico, E., \& à Pesquisa, V. E. D. I. Relação Dos Nutrientes Com A Ansiedade E Depressão.

dos Santos TAVARES, D., FlAMINI, D. T., \& de SOUZA, R. M. (2014). Nutrição No Tratamento Dos Processos Depressivos. Revista Funec CientíficaNutrição-Sem Circulação, 2(3), 32-45.

Fernstrom, JD (2016). Uma perspectiva sobre a segurança do triptofano suplementar com base em seus destinos metabólicos. The Journal of Nutrition, 146 (12), 2601S-2608S.

Fernstrom, J. D., Langham, K. A., Marcelino, L. M., Irvine, Z. L., Fernstrom, M. H., \& Kaye, W. H. (2013). A ingestão de diferentes proteínas dietéticas por humanos induz grandes mudanças na proporção de triptofano plasmático, um preditor da captação de triptofano pelo cérebro e da síntese de serotonina. Nutrição clínica , 32 (6), 1073-1076.

Ferraz, S. D. O. (2021). Alimentação Como Auxiliar Na Recuperação Pós-Covid 19: Diet as an Aid in Post-Covid Recovery 19. CPAH Scientific Journal of Health, 1(2), 40-46.

Jacka, F. N., Cherbuin, N., Anstey, K. J., \& Butterworth, P. (2015). Does reverse causality explain the relationship between diet and depression? Journal of affective disorders, $175,248-250$.

Jacobs, B. L., Martín-Cora, F. J., \& Fornal, C. A. (2002). Activity of medullary serotonergic neurons in freely moving animals. Brain Research Reviews, 40(13), 45-52.

Jenkins, T. A., Nguyen, J. C., Polglaze, K. E., \& Bertrand, P. P. (2016). Influence of tryptophan and serotonin on mood and cognition with a possible role of the gut-brain axis. Nutrients, $8(1), 56$.

Kennedy, D. O. (2016). B vitamins and the brain: mechanisms, dose and efficacy — a review. Nutrients, 8(2), 68 .

Lima, C. L. S., Lira, S. M., Holanda, M. O., da Silva, J. Y. G., Moura, V. B., Oliveira, J. D. S. M., \& Guedes, M. I. F. (2020). Bases fisiológicas e medicamentosas do transtorno da ansiedade. Research, Society and Development, 9(9), e808997780-e808997780.

Lindseth, G., Helland, B., \& Caspers, J. (2015). The effects of dietary tryptophan on affective disorders. Archives of psychiatric nursing, 29 (2), $102-107$.

Machado, A. C. P., dos Santos Souza, N. E. C., de Medeiros, V. G., dos Anjos Queiroz, C. R. A., \& Boff, T. (2018). Alimentos Ricos Em Triptofano: Segredo Da Felicidade? Ciência Viva. Mg.

Mazzini, M. C. R., Grossi, M., \& Malheiros, S. V. P. (2013). Regulação nutricional e neuroendócrina da serotonina podem influenciar a síndrome pré menstrual. Perspectivas Médicas, 24(1), 43-50.

Mendes, K. D. S., Silveira, R. C. C. P., \& Galvão, C. M. (2008). Integrative literature review: a research method to incorporate evidence in health care and nursing. Texto contexto enferm, 17(4), 758-64.

Moraes, R. W. D. (2014). Determinantes e construção do comportamento alimentar: uma revisão narrativa da literatura.

Santos, J. F. (2016). A influência da serotonina na fisiologia da depressão (Doctoral dissertation).

Setyarini, D., \& Santoso, B. W. (2020). The Effect of Giving Ambon Banana (Musa Paradisiaca, Sp) To Decrease of Anxiety Levels in Adult Scizofrenia. Executive Editor, 11(7), 1226.

Sezini, A. M., \& do Coutto Gil, C. S. G. (2014). Nutrientes e depressão. Vita et Sanitas, 8(1), 39-57.

Strasser, B., Gostner, J. M., \& Fuchs, D. (2016). Mood, food, and cognition: role of tryptophan and serotonin. Current Opinion in Clinical Nutrition \& Metabolic Care, 19(1), 55-61.

Szeitz, A., \& Bandiera, S. M. (2018). Analysis and measurement of serotonin. Biomedical Chromatography, $32(1)$, e4135.

Watts, S. W., Morrison, S. F., Davis, R. P., \& Barman, S. M. (2012). Serotonin and blood pressure regulation. Pharmacological reviews, 64(2), 359-388.

Zanello, D. R. D. P. (2012). Efeitos do L-Triptofano sobre ansiedade, compulsão e escolha alimentar. 